\title{
Iodine-129 concentration in seawater near Fukushima before and after the accident at the Fukushima Daiichi Nuclear Power Plant
}

\author{
T. Suzuki ${ }^{1}$, S. Otosaka ${ }^{1}$, J. Kuwabara ${ }^{2}$, H. Kawamura ${ }^{1}$, and T. Kobayashi ${ }^{1}$ \\ ${ }^{1}$ Japan Atomic Energy Agency, Ibaraki, Japan \\ ${ }^{2}$ Japan Atomic Energy Agency, Aomori, Japan \\ Correspondence to: T. Suzuki (suzuki.takashi58@jaea.go.jp)
}

Received: 26 November 2012 - Published in Biogeosciences Discuss.: 29 January 2013

Revised: 8 May 2013 - Accepted: 14 May 2013 - Published: 14 June 2013

\begin{abstract}
Anthropogenic radionuclides were released into the environment in large quantities by the Fukushima Daiichi Nuclear Power Plant (1FNPP) accident. To evaluate accident-derive ${ }^{129} \mathrm{I}$, the ${ }^{129} \mathrm{I}$ concentrations in seawater before and after the accident were compared.

Before the accident (2008-2009), the ${ }^{129}$ I concentrations in the western margin of the North Pacific between $32^{\circ} \mathrm{N}$ and $44^{\circ} \mathrm{N}$ showed a latitudinal gradient that was expressed as a linear function of latitude. The highest and average ${ }^{129} \mathrm{I}$ concentrations after the accident were 73 times and approximately 8 times, respectively, higher than those before the accident in this study area. Considering the distribution of ${ }^{129} \mathrm{I}$ in surface seawater, the accident-derived ${ }^{129} \mathrm{I}$ in the southern and northern stations of the 1FNPP was predominantly supplied by seawater advection and atmospheric deposition (including microbial volatilization), respectively.

As of October 2011, depth profiles of ${ }^{129}$ I revealed that ${ }^{129}$ I originating from the 1FNPP existed mainly in the upper $100 \mathrm{~m}$ depth. From the depth profiles, the cumulative inventories of accident-derived ${ }^{129} \mathrm{I}$ were estimated to be $(1.6-9.6) \times 10^{12}$ atoms $\mathrm{m}^{-2}$ in this study area.

On the basis of the ${ }^{129} \mathrm{I}$ data in the seawater near Fukushima, the effective dose of ${ }^{129}$ I from seafood ingestion was much smaller than the annual dose limit.
\end{abstract}

\section{Introduction}

Significant fission products such as ${ }^{134} \mathrm{Cs}$ (half-life: $2.06 \mathrm{yr}$ ), ${ }^{137} \mathrm{Cs}$ (half-life: $30.2 \mathrm{yr}$ ), and ${ }^{131} \mathrm{I}$ (half-life: 8.02 days) were released into the environment by the Fukushima Daiichi Nuclear Power Plant (1FNPP) accident caused by the Great East
Japan earthquake and tsunami on 11 March 2011 (Honda et al., 2012; Momoshima et al., 2012). From the viewpoint of environmental safety with respect to nuclear accidents, ${ }^{134} \mathrm{Cs},{ }^{137} \mathrm{Cs}$, and ${ }^{131} \mathrm{I}$ are regarded as important radionuclides because of their high fission yield and high $\gamma$-ray energy.

Radioiodine can enter the human body via food and drinking water and then selectively accumulate in the thyroid, creating an irradiation risk. A significantly higher incidence of thyroid cancer in children living around the Chernobyl accident site has been reported (Baverstock et al., 1992). Although thyroid cancer related to ${ }^{131} \mathrm{I}$ exposure as a result of nuclear accidents has been of considerable concern, less attention has been paid to the increasing inventory of ${ }^{129} \mathrm{I}$ (half-life: $1.57 \times 10^{7} \mathrm{yr}$ ) from the 1FNPP accident. Because ${ }^{129}$ I has a long half-life, the amount and behavior of accidentderived ${ }^{129} \mathrm{I}$ in the environment should be investigated to address concerns about the radiological impacts to future generations. Measuring ${ }^{129} \mathrm{I}$ in environmental samples also offers an opportunity to retrospectively analyze the migration of accident-derived ${ }^{131} \mathrm{I}$, which has decayed and become undetectable from the passage of post-accident time.

Anthropogenic ${ }^{129} \mathrm{I}$ is released into the environment from nuclear fuel reprocessing plants and is then transported mainly to the northern hemisphere (Suzuki et al., 2010; Snyder et al., 2010; Toyama et al., 2012). Thus, the ${ }^{129} \mathrm{I}$ level in the environment is already increased from its natural level. Soil and seawater play a role as a reservoir of ${ }^{129} \mathrm{I}(\mathrm{Mu}-$ ramatsu et al., 2004). The impact of ${ }^{129}$ I on land from the 1FNPP accident has been reported (Miyake et al., 2012), but not the impact on the ocean. This study focuses on the impact of ${ }^{129} \mathrm{I}$ on seawater from the $1 \mathrm{FNPP}$ accident. To precisely 


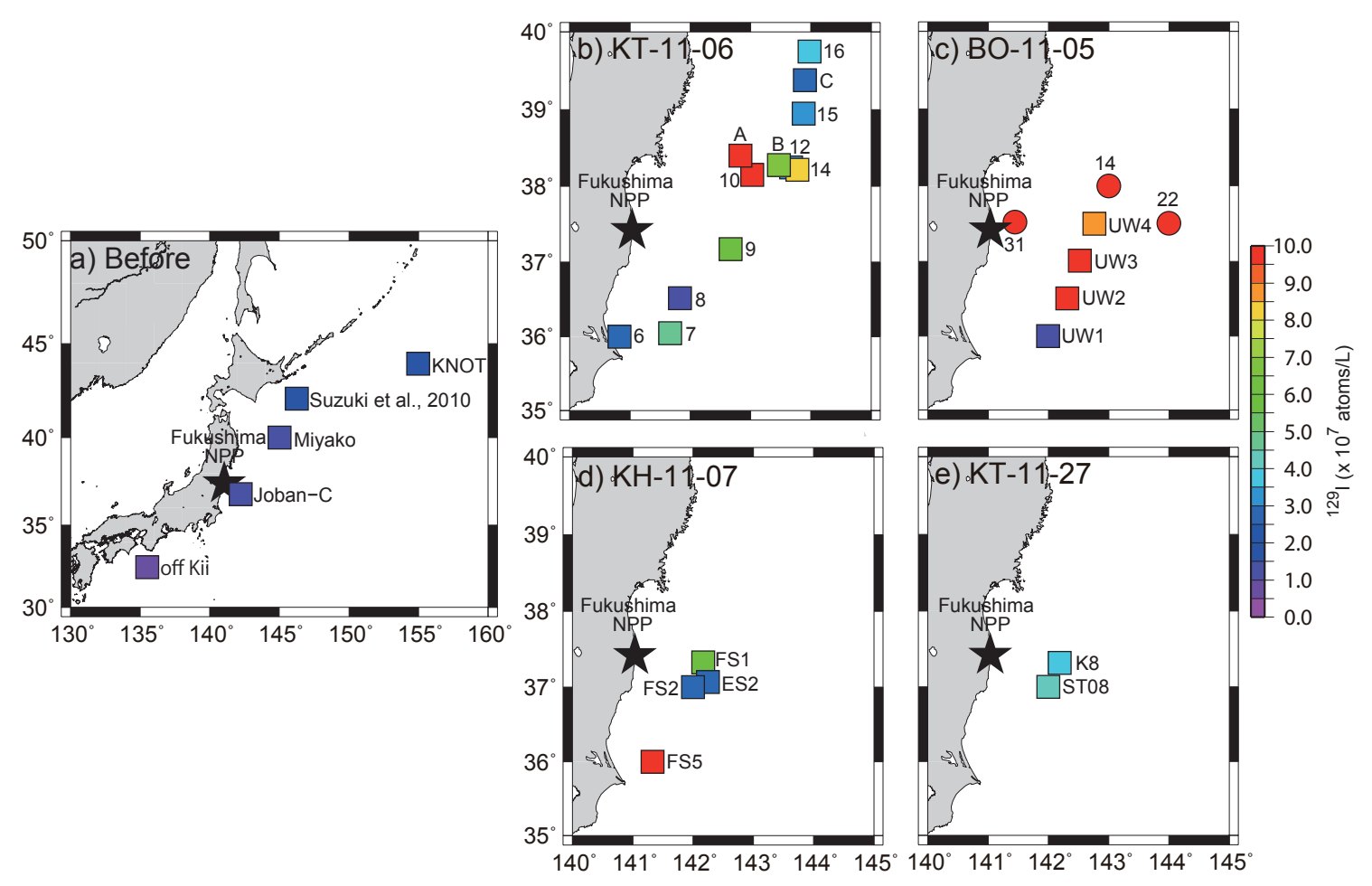

Fig. 1. Map of sampling locations and the result of surface ${ }^{129}$ I concentrations before the 1FNPP accident (a) and afterwards (b-e). After the accident, seawater sampling was undertaken during four cruises: (b) KT-11-06, (c) BO-11-05, (d) KH-11-07, and (e) KT-11-27. In (c), circle symbols were cited from Hou et al. (2013). Red at the sampling locations indicates that the ${ }^{129} \mathrm{I}$ concentration is higher than $10 \times 10^{7}$ atoms $\mathrm{L}^{-1}$.

evaluate the increase of ${ }^{129}$ I caused by the accident, information about the ${ }^{129} \mathrm{I}$ before the accident in the western North Pacific Ocean is crucial. Many studies about ${ }^{129} \mathrm{I}$ in seawater have been conducted around nuclear fuel reprocessing plants (Keogh et al., 2007; Raisbeck and Yiou, 1999; Alfimov et al., 2004; Michel et al., 2012; Hou et al., 2001), but only a few studies have been undertaken in the Pacific Ocean (Suzuki et al., 2010; Povinec et al., 2010). Therefore, the background level of ${ }^{129} \mathrm{I}$ in the western North Pacific Ocean had not previously been determined.

Prior to the 1FNPP accident, a new nuclear fuel reprocessing plant was tested for routine operation at Rokkasho, Japan. Anticipating the release of ${ }^{129} \mathrm{I}$ from the operation of the reprocessing plant, seawater samples in the western North Pacific Ocean were collected to clearly define the background level before plant operations commenced. After the 1FNPP accident, seawater samples collected before the accident proved useful for evaluating the background level of ${ }^{129} \mathrm{I}$ in the western North Pacific Ocean. In this paper, we report the distributions of ${ }^{129} \mathrm{I}$ in the western North Pacific before and after the 1FNPP accident and evaluate the accident-derived ${ }^{129} \mathrm{I}$ in seawater. In addition, we infer the migration of ${ }^{129} \mathrm{I}$ off Fukushima after the accident and assess the internal dose of accident-related ${ }^{129} \mathrm{I}$ as a result of seafood consumption.

\section{Experimental}

\subsection{Seawater sampling}

Seawater sampling before the 1FNPP accident were conducted at four stations: KNOT $\left(154^{\circ} 58^{\prime} \mathrm{E}, 43^{\circ} 58^{\prime} \mathrm{N}\right)$ on 10 May 2008 by the T/S Oshoro Maru; off Kii $\left(135^{\circ} 31^{\prime}\right.$ E, $\left.32^{\circ} 28^{\prime} \mathrm{N}\right)$ on 5 August 2008 by the R/V Soyo Maru; Joban C $\left(142^{\circ} 13^{\prime} \mathrm{E}, 36^{\circ} 48^{\prime} \mathrm{N}\right)$ on 18 July 2009 by the R/V Soyo Maru; and Miyako $\left(145^{\circ} 00^{\prime} \mathrm{E}, 40^{\circ} 00^{\prime} \mathrm{N}\right)$ on 20 July 2009 by the R/V Soyo Maru, as shown in Fig. 1a. Figure 1a includes another station that can provide a background level for 1FNPP in the western North Pacific Ocean (Suzuki et al., 2010).

Seawater sampling after the 1FNPP accident were undertaken by four expeditions: from 27 April to 1 May 2011 by the R/V Tansei Maru, cruise KT-11-06 (Fig. 1b); from 12 to 13 June 2011 by the R/V Bosei-Maru, cruise BO-11-05 (Fig. 1c); from 2 to 3 August 2011 by the R/V Hakuho Maru, cruise KH-11-07 (Fig. 1d); and on 29 October 2011 by the R/V Tansei Maru, cruise KT-11-27 (Fig. 1e). Seawater samples at eight stations were collected from surface to deep layers at stations A, B, and C by cruise KT-11-06; at stations FS1, ES2, and FS2 by cruise KH-11-07; and at stations ST08 and K8 by cruise KT-11-27. Fourteen surface seawater samples were collected at other stations. 


\subsection{Analytical procedure}

Iodine was extracted from seawater samples by the solvent extraction technique (Suzuki et al., 2008). Ascorbic acid with hydrochloric acid was added to a seawater sample to reduce iodate to iodide after passing through a $0.45 \mu \mathrm{m}$ filter. Iodide was oxidized to molecular iodine by the addition of sodium nitrite for the extraction to chloroform. Back extraction was carried out from chloroform using sodium sulfite by reducing molecular iodine to iodide. The extracted iodide was precipitated as silver iodide by silver nitrate. The silver iodide sample was washed by nitric acid and pure water and then loaded to a copper target holder.

Iodine isotopic ratios were measured by accelerator mass spectrometry at the Aomori Research and Development Center of the Japan Atomic Energy Agency. All measured data was normalized to the standard reference material having ${ }^{129} \mathrm{I} /{ }^{127} \mathrm{I}=(9.85 \pm 0.12) \times 10^{-13}$, which was obtained from the National Institute of Standards and Technology (NIST SRM 3230) (Suzuki et al., 2006).

Total iodine (iodate + iodide) concentrations in seawater samples were measured by cathodic stripping square wave voltammetry or quadrupole inductively coupled plasma mass spectrometry (Campos, 1997).

\section{Results and discussion}

\subsection{Distribution of ${ }^{129} \mathrm{I}$ in surface seawater}

\subsubsection{Before the 1FNPP accident}

With regard to the global distribution of ${ }^{129} \mathrm{I}$ in surface reservoirs, the latitudinal distribution provides us with information useful for understanding the fate of ${ }^{129} \mathrm{I}$ (Snyder et al., 2010). Thus, the ${ }^{129}$ I concentrations in surface seawater before and after the 1FNPP accident with another published data (Hou et al., 2013) are plotted in Fig. 2 against the latitude of the sampling location. The surface ${ }^{129} \mathrm{I}$ concentrations are also plotted at the sampling locations in Fig. 1.

The concentration of ${ }^{129} \mathrm{I}$ before the 1FNPP accident was in the 0.94 (off Kii) -1.83 (offshore of Kushiro: Suzuki et al., 2010) $\times 10^{7}$ atoms $\mathrm{L}^{-1}$ range (Table 1 ). The ${ }^{129} \mathrm{I}$ in surface seawater between $32^{\circ} \mathrm{N}$ and $44^{\circ} \mathrm{N}$ in the western North Pacific Ocean before the 1FNPP accident was high in the north and decreased with decreasing latitude. These concentrations correspond to the locations of nuclear fuel reprocessing plants, which is a primary source of ${ }^{129} \mathrm{I}$ in the environment. Since major reprocessing plants such as Sellafield $\left(54^{\circ} 00^{\prime} \mathrm{N}\right)$ in the United Kingdom, La Hague $\left(49^{\circ} 30^{\prime} \mathrm{N}\right)$ in France, and Hanford $\left(46^{\circ} 37^{\prime} \mathrm{N}\right)$ in the United States are located in the middle- to high-latitude regions of the Northern Hemisphere, ${ }^{129}$ I originating from nuclear fuel reprocessing plants was mainly distributed over the Northern Hemisphere (Snyder et al., 2010; Suzuki et al., 2010; Moran et al., 1999). The latitudinal distribution of ${ }^{129}$ I in surface seawater before

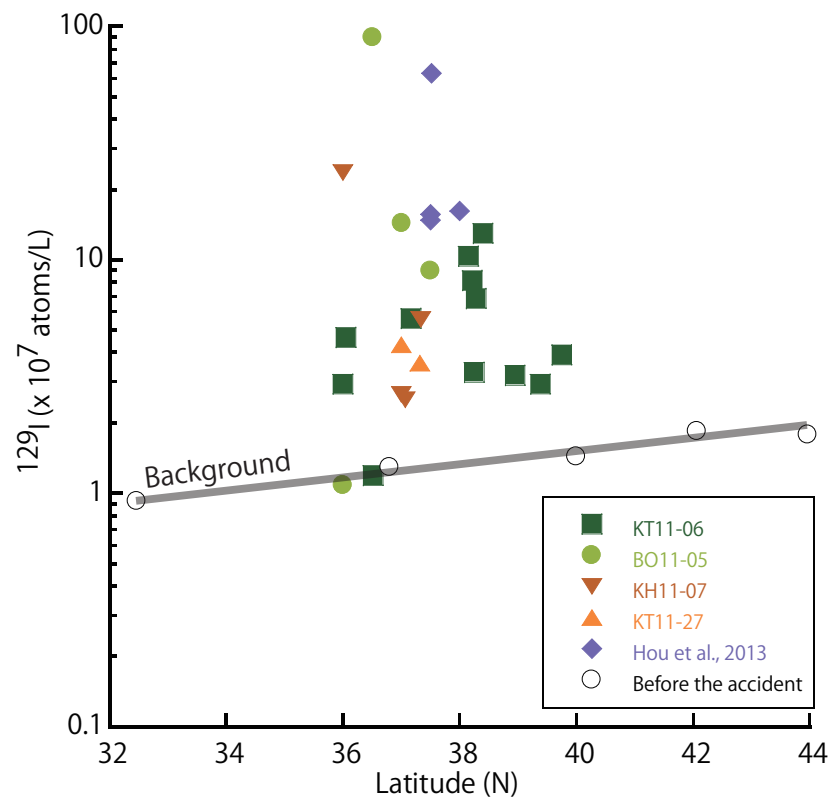

Fig. 2. ${ }^{129} \mathrm{I}$ concentrations in surface seawater before and after the 1FNPP accident as a function of latitude. The dark green, light green, dark orange, and light orange symbols indicate cruises KT-11-06, BO-11-05, KH11-07, and KT-11-27, respectively, after the 1FNPP accident. The purple symbols were cited from Hou et al. (2013). The white symbols indicate a cruise before the Fukushima NPP accident.

the 1FNPP accident is attributed to the atmospheric deposition of ${ }^{129} \mathrm{I}$ originating from the major reprocessing plants and can be expressed as a linear function of latitude (Fig. 2).

${ }^{129} \mathrm{C}_{\mathrm{BG}}=0.080 \times L-1.7,(32<L<44)$,

where ${ }^{129} \mathrm{C}_{\mathrm{BG}}\left(10^{7}\right.$ atoms $\left.\mathrm{L}^{-1}\right)$ is the ${ }^{129} \mathrm{I}$ concentration in surface seawater before the 1FNPP accident and $L\left({ }^{\circ} \mathrm{N}\right)$ is the latitude.

\subsubsection{After the 1FNPP accident}

In this study area, the ${ }^{129} \mathrm{I}$ concentrations in the surface seawater after the 1FNPP accident were in the $1.08-89.8 \times 10^{7}$ atoms $\mathrm{L}^{-1}$ (corresponding to ${ }^{129} \mathrm{I} /{ }^{127} \mathrm{I}=4.47-362 \times 10^{-11}$ ) range (Figs. 1 and 2 ). The highest ${ }^{129} \mathrm{I}$ concentration $\left({ }^{129} \mathrm{I}=89.8 \times 10^{7}\right.$ atoms $\mathrm{L}^{-1}$, ${ }^{129} \mathrm{I} /{ }^{127} \mathrm{I}=362 \times 10^{-11}$ ) was observed at station UW2 on 12 June 2011 during cruise BO-11-05, this value was 73 times higher than that before the accident, and this value was higher than that of the previous study (Hou et al., 2013). The average ${ }^{129}$ I concentration in surface seawater after the 1 FNPP accident was $9.3 \times 10^{7}$ atoms $\mathrm{L}^{-1}$, a value approximately 8 times higher than that before the accident. Since routine operation of the Tokai reprocessing plant and test operation of the Rokkasho reprocessing plant had both ceased in 2008 , the elevated ${ }^{129} \mathrm{I}$ can be considered to originate from 1FNPP. Accident-derived ${ }^{129} \mathrm{I}$ was detected 
Table 1. Concentrations of ${ }^{129} \mathrm{I}$ in seawater samples.

\begin{tabular}{|c|c|c|c|c|c|c|c|c|c|c|c|}
\hline Cruise & Station & Date & $\mathrm{E}$ & $\mathrm{N}$ & $\begin{array}{l}\text { Depth } \\
\text { (m) }\end{array}$ & $\begin{array}{c}\text { Salinity } \\
\text { PSU }\end{array}$ & $\begin{array}{l}\text { Potential } \\
\text { Temp. } \\
{ }^{\circ} \mathrm{C}\end{array}$ & $\begin{array}{c}\text { Density } \\
\sigma_{\theta}\end{array}$ & $\begin{array}{l}{ }^{129} \mathrm{I} /{ }^{127} \mathrm{I} \\
\times 10^{-11}\end{array}$ & $\times 10^{7}$ atoms $\mathrm{L}^{-1}$ & $\mathrm{nBqL}^{-1}$ \\
\hline Before & e $1 \mathrm{FNPP}$ & ccident & & & & & & & & & \\
\hline OS08 & KNOT & 10 May 2008 & $154^{\circ} 58^{\prime}$ & $43^{\circ} 58^{\prime}$ & 4 & 33.178 & 5.477 & 26.176 & $5.85 \pm 0.21$ & $1.78 \pm 0.07$ & $24.9 \pm 1.0$ \\
\hline & & & & & 10 & 33.178 & 5.474 & 26.176 & $6.22 \pm 0.22$ & $1.83 \pm 0.08$ & $25.6 \pm 1.1$ \\
\hline & & & & & 20 & 33.217 & 5.339 & 26.223 & $6.25 \pm 0.19$ & $1.78 \pm 0.06$ & $24.9 \pm 0.8$ \\
\hline & & & & & 30 & 33.289 & 5.084 & 26.309 & $5.53 \pm 0.25$ & $1.56 \pm 0.08$ & $21.8 \pm 1.2$ \\
\hline & & & & & 39 & 33.400 & 4.717 & 26.438 & $6.38 \pm 0.23$ & $1.72 \pm 0.07$ & $24.1 \pm 1.0$ \\
\hline & & & & & 48 & 33.428 & 4.661 & 26.467 & $5.70 \pm 0.24$ & $1.63 \pm 0.09$ & $22.7 \pm 1.2$ \\
\hline & & & & & 74 & 33.572 & 5.079 & 26.534 & $4.79 \pm 0.21$ & $1.53 \pm 0.08$ & $21.4 \pm 1.0$ \\
\hline & & & & & 99 & 33.563 & 4.544 & 26.586 & $5.70 \pm 0.20$ & $1.63 \pm 0.07$ & $22.8 \pm 0.9$ \\
\hline & & & & & 122 & 33.531 & 3.930 & 26.625 & $5.31 \pm 0.18$ & $1.47 \pm 0.05$ & $20.5 \pm 0.7$ \\
\hline & & & & & 149 & 33.512 & 3.116 & 26.687 & $4.42 \pm 0.23$ & $1.32 \pm 0.08$ & $18.4 \pm 1.1$ \\
\hline & & & & & 197 & 33.637 & 2.859 & 26.810 & $3.98 \pm 0.17$ & $1.18 \pm 0.06$ & $16.5 \pm 0.8$ \\
\hline & & & & & 299 & 33.869 & 3.191 & 26.965 & $2.65 \pm 0.12$ & $0.83 \pm 0.04$ & $11.7 \pm 0.6$ \\
\hline & & & & & 396 & 33.991 & 3.181 & 27.063 & $2.06 \pm 0.10$ & $0.66 \pm 0.04$ & $9.2 \pm 0.5$ \\
\hline & & & & & 495 & 34.118 & 3.279 & 27.155 & $1.45 \pm 0.09$ & $0.44 \pm 0.03$ & $6.2 \pm 0.4$ \\
\hline & & & & & 743 & 34.309 & 2.842 & 27.348 & $0.56 \pm 0.06$ & $0.17 \pm 0.02$ & $2.3 \pm 0.3$ \\
\hline & & & & & 990 & 34.414 & 2.528 & 27.459 & $0.22 \pm 0.05$ & $0.07 \pm 0.01$ & $1.0 \pm 0.2$ \\
\hline & & & & & 1235 & 34.462 & 2.271 & 27.519 & $0.19 \pm 0.04$ & $0.06 \pm 0.01$ & $0.8 \pm 0.2$ \\
\hline & & & & & 1483 & 34.510 & 2.079 & 27.573 & $0.07 \pm 0.05$ & $0.02 \pm 0.02$ & $0.3 \pm 0.2$ \\
\hline & & & & & 1727 & 34.559 & 1.895 & 27.626 & $0.03 \pm 0.05$ & $0.01 \pm 0.01$ & $0.1 \pm 0.2$ \\
\hline & & & & & 1974 & 34.592 & 1.722 & 27.666 & $0.03 \pm 0.04$ & $0.01 \pm 0.01$ & $0.1 \pm 0.2$ \\
\hline & & & & & 2708 & 34.642 & 1.390 & 27.730 & $0.03 \pm 0.04$ & $0.01 \pm 0.01$ & $0.1 \pm 0.2$ \\
\hline & & & & & 2955 & 34.651 & 1.320 & 27.743 & $0.01 \pm 0.04$ & $0.01 \pm 0.01$ & $0.1 \pm 0.2$ \\
\hline SY08 & off Kii & 5 Aug 2008 & $135^{\circ} 31^{\prime}$ & $32^{\circ} 28^{\prime}$ & 6 & 34.081 & 29.931 & 21.061 & $3.13 \pm 0.12$ & $0.94 \pm 0.04$ & $13.2 \pm 0.6$ \\
\hline & & & & & 50 & 34.309 & 25.889 & 22.547 & $2.84 \pm 0.11$ & $0.86 \pm 0.04$ & $12.0 \pm 0.5$ \\
\hline & & & & & 101 & 34.796 & 21.571 & 24.133 & $3.74 \pm 0.15$ & $1.12 \pm 0.05$ & $15.7 \pm 0.8$ \\
\hline & & & & & 151 & 34.828 & 19.726 & 24.703 & $3.09 \pm 0.12$ & $0.92 \pm 0.04$ & $12.9 \pm 0.6$ \\
\hline & & & & & 201 & 34.810 & 18.907 & 24.900 & $3.42 \pm 0.11$ & $1.08 \pm 0.04$ & $15.1 \pm 0.6$ \\
\hline & & & & & 251 & 34.800 & 18.379 & 25.025 & $3.84 \pm 0.14$ & $1.17 \pm 0.05$ & $16.3 \pm 0.7$ \\
\hline & & & & & 498 & 34.435 & 12.659 & 26.023 & $3.88 \pm 0.12$ & $1.23 \pm 0.05$ & $17.2 \pm 0.7$ \\
\hline & & & & & 750 & 34.255 & 6.187 & 26.940 & $1.59 \pm 0.13$ & $0.52 \pm 0.05$ & $7.3 \pm 0.7$ \\
\hline & & & & & 999 & 34.388 & 3.752 & 27.324 & $0.85 \pm 0.07$ & $0.27 \pm 0.02$ & $3.7 \pm 0.3$ \\
\hline & & & & & 1248 & 34.460 & 2.931 & 27.461 & $0.60 \pm 0.06$ & $0.20 \pm 0.02$ & $2.8 \pm 0.3$ \\
\hline & & & & & 1499 & 34.522 & 2.480 & 27.550 & $0.53 \pm 0.05$ & $0.17 \pm 0.02$ & $2.4 \pm 0.2$ \\
\hline & & & & & 2002 & 34.599 & 1.883 & 27.660 & $0.84 \pm 0.08$ & $0.27 \pm 0.03$ & $3.7 \pm 0.4$ \\
\hline SY09 & Joban C & 18 Jul 2009 & $142^{\circ} 13^{\prime}$ & $36^{\circ} 48^{\prime}$ & 5 & 34.110 & 21.625 & 23.648 & $4.75 \pm 0.28$ & $1.29 \pm 0.08$ & $18.1 \pm 1.1$ \\
\hline & & & & & 10 & 34.201 & 20.819 & 23.937 & $4.30 \pm 0.25$ & $1.24 \pm 0.07$ & $17.3 \pm 1.1$ \\
\hline & & & & & 20 & 34.142 & 16.369 & 25.005 & $4.35 \pm 0.25$ & $1.19 \pm 0.07$ & $16.6 \pm 1.0$ \\
\hline & & & & & 29 & 34.319 & 13.446 & 25.777 & $4.10 \pm 0.26$ & $1.20 \pm 0.08$ & $16.7 \pm 1.1$ \\
\hline & & & & & 39 & 34.267 & 12.366 & 25.951 & $4.47 \pm 0.26$ & $1.29 \pm 0.08$ & $18.1 \pm 1.1$ \\
\hline & & & & & 50 & 34.243 & 11.561 & 26.085 & $3.97 \pm 0.25$ & $1.13 \pm 0.07$ & $15.8 \pm 1.0$ \\
\hline & & & & & 69 & 34.135 & 10.302 & 26.227 & $5.22 \pm 0.29$ & $1.47 \pm 0.08$ & $20.5 \pm 1.1$ \\
\hline & & & & & 100 & 33.979 & 8.711 & 26.366 & $4.73 \pm 0.28$ & $1.42 \pm 0.09$ & $19.8 \pm 1.2$ \\
\hline & & & & & 124 & 34.003 & 8.420 & 26.430 & $4.66 \pm 0.27$ & $1.32 \pm 0.08$ & $18.5 \pm 1.1$ \\
\hline & & & & & 149 & 33.842 & 7.167 & 26.486 & $5.19 \pm 0.24$ & $1.48 \pm 0.07$ & $20.7 \pm 1.0$ \\
\hline & & & & & 198 & 33.685 & 5.027 & 26.631 & $5.42 \pm 0.28$ & $1.49 \pm 0.08$ & $20.9 \pm 1.1$ \\
\hline & & & & & 298 & 33.718 & 3.908 & 26.778 & $4.33 \pm 0.25$ & $1.31 \pm 0.08$ & $18.3 \pm 1.1$ \\
\hline & & & & & 397 & 33.954 & 4.230 & 26.934 & $2.68 \pm 0.20$ & $0.83 \pm 0.06$ & $11.5 \pm 0.9$ \\
\hline & & & & & 496 & 34.093 & 4.095 & 27.059 & $1.65 \pm 0.16$ & $0.51 \pm 0.05$ & $7.1 \pm 0.7$ \\
\hline & & & & & 743 & 34.229 & 3.373 & 27.296 & $0.70 \pm 0.14$ & $0.21 \pm 0.04$ & $3.0 \pm 0.6$ \\
\hline & & & & & 990 & 34.410 & 2.787 & 27.439 & $0.09 \pm 0.11$ & $0.03 \pm 0.04$ & $0.4 \pm 0.5$ \\
\hline & & & & & 1236 & 34.476 & 2.397 & 27.527 & $0.04 \pm 0.10$ & $0.01 \pm 0.03$ & $0.2 \pm 0.4$ \\
\hline & & & & & 1730 & 34.556 & 1.992 & 27.625 & $0.18 \pm 0.11$ & $0.06 \pm 0.03$ & $0.8 \pm 0.5$ \\
\hline & & & & & 1976 & 34.586 & 1.814 & 27.664 & $0.03 \pm 0.10$ & $0.01 \pm 0.03$ & $0.1 \pm 0.4$ \\
\hline & & & & & 2221 & 34.608 & 1.674 & 27.694 & $0.02 \pm 0.10$ & $0.01 \pm 0.03$ & $0.1 \pm 0.5$ \\
\hline & Miyako & $20 \mathrm{Jul} 2009$ & $145^{\circ} 00^{\prime}$ & $40^{\circ} 00^{\prime}$ & 5 & 33.817 & 17.583 & 24.469 & $5.01 \pm 0.25$ & $1.43 \pm 0.07$ & $19.9 \pm 1.0$ \\
\hline & & & & & 10 & 33.815 & 17.586 & 24.467 & $4.52 \pm 0.24$ & $1.28 \pm 0.07$ & $17.9 \pm 1.0$ \\
\hline & & & & & 20 & 33.863 & 15.008 & 25.095 & $5.12 \pm 0.28$ & $1.36 \pm 0.08$ & $19.0 \pm 1.1$ \\
\hline & & & & & 30 & 34.047 & 13.912 & 25.471 & $5.41 \pm 0.27$ & $1.53 \pm 0.08$ & $21.4 \pm 1.1$ \\
\hline & & & & & 39 & 34.166 & 14.011 & 25.542 & $4.48 \pm 0.24$ & $1.25 \pm 0.07$ & $17.5 \pm 1.0$ \\
\hline & & & & & 50 & 34.273 & 13.041 & 25.823 & $4.73 \pm 0.26$ & $1.34 \pm 0.08$ & $18.8 \pm 1.1$ \\
\hline & & & & & 74 & 34.188 & 11.315 & 26.088 & $5.13 \pm 0.28$ & $1.46 \pm 0.08$ & $20.4 \pm 1.1$ \\
\hline & & & & & 99 & 33.785 & 8.127 & 26.303 & $5.42 \pm 0.30$ & $1.46 \pm 0.08$ & $20.5 \pm 1.1$ \\
\hline
\end{tabular}


Table 1. Continued.

\begin{tabular}{|c|c|c|c|c|c|c|c|c|c|c|c|}
\hline \multirow[t]{2}{*}{ Cruise } & \multirow[t]{2}{*}{ Station } & \multirow[t]{2}{*}{ Date } & \multirow[b]{2}{*}{$\mathrm{E}$} & \multirow[b]{2}{*}{$\mathrm{N}$} & \multirow{2}{*}{$\begin{array}{c}\text { Depth } \\
\text { (m) }\end{array}$} & \multirow{2}{*}{$\begin{array}{c}\text { Salinity } \\
\text { PSU }\end{array}$} & \multirow{2}{*}{$\begin{array}{l}\text { Potential } \\
\text { Temp. } \\
{ }^{\circ} \mathrm{C}\end{array}$} & \multirow{2}{*}{$\begin{array}{c}\text { Density } \\
\sigma_{\theta}\end{array}$} & \multirow{2}{*}{$\begin{array}{l}{ }^{129} \mathrm{I} /{ }^{127} \mathrm{I} \\
\times 10^{-11}\end{array}$} & \multicolumn{2}{|c|}{${ }^{129} \mathrm{I}$} \\
\hline & & & & & & & & & & $\times 10^{7}$ atoms $\mathrm{L}^{-1}$ & $\mathrm{nBq} \mathrm{L}^{-1}$ \\
\hline & & & & & 123 & 33.611 & 6.232 & 26.427 & $4.38 \pm 0.24$ & $1.27 \pm 0.07$ & $17.8 \pm 1.0$ \\
\hline & & & & & 148 & 33.612 & 5.608 & 26.505 & $6.19 \pm 0.30$ & $1.72 \pm 0.09$ & $24.1 \pm 1.2$ \\
\hline & & & & & 199 & 33.878 & 6.483 & 26.607 & $4.27 \pm 0.24$ & $1.21 \pm 0.07$ & $17.0 \pm 1.0$ \\
\hline & & & & & 298 & 33.847 & 4.663 & 26.802 & $3.81 \pm 0.20$ & $1.09 \pm 0.06$ & $15.2 \pm 0.8$ \\
\hline & & & & & 397 & 33.907 & 4.330 & 26.885 & $3.03 \pm 0.21$ & $0.08 \pm 0.06$ & $12.3 \pm 0.9$ \\
\hline & & & & & 742 & 34.285 & 3.421 & 27.280 & $0.97 \pm 0.14$ & $0.30 \pm 0.04$ & $4.2 \pm 0.6$ \\
\hline & & & & & 1482 & 34.518 & 2.177 & 27.580 & $0.15 \pm 0.10$ & $0.05 \pm 0.03$ & $0.7 \pm 0.4$ \\
\hline & & & & & 1728 & 34.561 & 1.955 & 27.632 & $0.07 \pm 0.10$ & $0.02 \pm 0.03$ & $0.3 \pm 0.4$ \\
\hline After the 1 & NPP acci & & & & & & & & & & \\
\hline KT11-06 & 6 & 27 Apr 2011 & $140^{\circ} 50^{\prime}$ & $36^{\circ} 00^{\prime}$ & 0 & & & & $11.49 \pm 0.45$ & $2.93 \pm 0.11$ & $41.0 \pm 1.6$ \\
\hline & 7 & 28 Apr 2011 & $141^{\circ} 40^{\prime}$ & $36^{\circ} 03^{\prime}$ & 0 & & & & $18.88 \pm 0.51$ & $4.65 \pm 0.13$ & $65.1 \pm 1.8$ \\
\hline & 8 & 28 Apr 2011 & $141^{\circ} 50^{\prime}$ & $36^{\circ} 31^{\prime}$ & 0 & & & & $4.70 \pm 0.29$ & $1.19 \pm 0.07$ & $16.7 \pm 1.0$ \\
\hline & 9 & 29 Apr 2011 & $142^{\circ} 40^{\prime}$ & $37^{\circ} 10^{\prime}$ & 0 & & & & $22.70 \pm 0.85$ & $5.60 \pm 0.21$ & $78.3 \pm 2.9$ \\
\hline & 10 & 29 Apr 2011 & $143^{\circ} 02^{\prime}$ & $38^{\circ} 09^{\prime}$ & 0 & & & & $42.01 \pm 0.92$ & $10.37 \pm 0.23$ & $145.0 \pm 3.2$ \\
\hline & 12 & 29 Apr 2011 & $143^{\circ} 40^{\prime}$ & $38^{\circ} 15^{\prime}$ & 0 & & & & $13.14 \pm 0.60$ & $3.29 \pm 0.15$ & $46.1 \pm 2.1$ \\
\hline & 14 & 30 Apr 2011 & $143^{\circ} 47^{\prime}$ & $38^{\circ} 13^{\prime}$ & 0 & & & & $32.49 \pm 0.81$ & $8.15 \pm 0.20$ & $114.0 \pm 2.8$ \\
\hline & $\mathrm{A}$ & 29 Apr 2011 & $142^{\circ} 50^{\prime}$ & $38^{\circ} 24^{\prime}$ & 9 & 33.988 & 11.262 & 25.941 & $50.78 \pm 0.96$ & $12.97 \pm 0.25$ & $181.4 \pm 3.4$ \\
\hline & & & & & 29 & 33.936 & 10.511 & 26.034 & $70.37 \pm 1.53$ & $17.65 \pm 0.38$ & $246.9 \pm 5.4$ \\
\hline & & & & & 49 & 34.252 & 10.403 & 26.229 & $30.92 \pm 0.88$ & $8.05 \pm 0.23$ & $112.6 \pm 3.2$ \\
\hline & & & & & 98 & 33.741 & 6.928 & 26.437 & $12.59 \pm 0.51$ & $3.18 \pm 0.13$ & $44.4 \pm 1.8$ \\
\hline & & & & & 198 & 33.701 & 5.484 & 26.589 & $5.89 \pm 0.32$ & $1.50 \pm 0.08$ & $21.0 \pm 1.1$ \\
\hline & & & & & 594 & 34.192 & 4.269 & 27.116 & $1.29 \pm 0.25$ & $0.34 \pm 0.07$ & $4.8 \pm 0.9$ \\
\hline & & & & & 1421 & 34.508 & 2.257 & 27.557 & $0.37 \pm 0.15$ & $0.10 \pm 0.04$ & $1.4 \pm 0.6$ \\
\hline & $\mathrm{B}$ & 30 Apr 2011 & $143^{\circ} 28^{\prime}$ & $38^{\circ} 17^{\prime}$ & 9 & 34.273 & 12.759 & 25.657 & $27.73 \pm 0.78$ & $6.83 \pm 0.20$ & $95.5 \pm 2.7$ \\
\hline & & & & & 50 & 34.274 & 11.513 & 25.894 & $11.64 \pm 0.56$ & $2.84 \pm 0.14$ & $39.7 \pm 1.9$ \\
\hline & & & & & 99 & 34.212 & 10.396 & 26.094 & $8.82 \pm 0.43$ & $2.22 \pm 0.11$ & $31.1 \pm 1.5$ \\
\hline & & & & & 198 & 33.987 & 8.294 & 26.435 & $5.66 \pm 0.41$ & $1.45 \pm 0.10$ & $20.2 \pm 1.5$ \\
\hline & & & & & 497 & 34.070 & 4.073 & 27.039 & $2.53 \pm 0.25$ & $0.69 \pm 0.07$ & $9.6 \pm 0.9$ \\
\hline & & & & & 989 & 34.375 & 2.932 & 27.392 & $0.91 \pm 0.23$ & $0.25 \pm 0.06$ & $3.5 \pm 0.9$ \\
\hline & $\mathrm{C}$ & 1 May 2011 & $143^{\circ} 54^{\prime}$ & $39^{\circ} 23^{\prime}$ & 10 & 33.957 & 9.981 & 26.165 & $11.63 \pm 0.69$ & $2.93 \pm 0.17$ & $40.9 \pm 2.4$ \\
\hline & & & & & 50 & 33.981 & 9.540 & 26.238 & $10.81 \pm 0.50$ & $2.73 \pm 0.13$ & $38.3 \pm 1.8$ \\
\hline & & & & & 99 & 33.985 & 9.199 & 26.294 & $11.70 \pm 0.57$ & $3.02 \pm 0.15$ & $42.2 \pm 2.1$ \\
\hline & 15 & 1 May 2011 & $143^{\circ} 53^{\prime}$ & $38^{\circ} 57^{\prime}$ & 0 & & & & $12.84 \pm 0.61$ & $3.19 \pm 0.15$ & $44.7 \pm 2.1$ \\
\hline & 16 & 1 May 2011 & $143^{\circ} 59^{\prime}$ & $39^{\circ} 45^{\prime}$ & 0 & & & & $15.84 \pm 0.54$ & $3.91 \pm 0.13$ & $54.7 \pm 1.9$ \\
\hline BO-11-05 & UW1 & 12 Jun 2011 & $141^{\circ} 59^{\prime}$ & $36^{\circ} 00^{\prime}$ & 0 & 34.379 & 21.299 & 23.643 & $4.47 \pm 0.27$ & $1.08 \pm 0.06$ & $15.2 \pm 0.9$ \\
\hline & UW2 & 12 Jun 2011 & $142^{\circ} 19^{\prime}$ & $36^{\circ} 31^{\prime}$ & 0 & 33.426 & 17.677 & 24.576 & $362.25 \pm 4.87$ & $89.76 \pm 1.21$ & $1255 \pm 16.9$ \\
\hline & UW3 & 12 Jun 2011 & $142^{\circ} 31^{\prime}$ & $37^{\circ} 01^{\prime}$ & 0 & 34.011 & 18.224 & 24.443 & $58.99 \pm 1.22$ & $14.36 \pm 0.30$ & $200.8 \pm 4.2$ \\
\hline & UW4 & 13 Jun 2011 & $142^{\circ} 45^{\prime}$ & $37^{\circ} 30^{\prime}$ & 0 & 34.108 & 18.947 & 24.262 & $36.36 \pm 0.75$ & $8.98 \pm 0.18$ & $125.6 \pm 2.6$ \\
\hline KH-11-07 & FS1 & 2 Aug 2011 & $142^{\circ} 10^{\prime}$ & $37^{\circ} 20^{\prime}$ & 0 & & & & $23.29 \pm 0.60$ & $5.56 \pm 0.14$ & $77.8 \pm 2.0$ \\
\hline & & & & & 101 & 33.772 & 8.277 & 26.437 & $7.33 \pm 0.33$ & $2.00 \pm 0.09$ & $28.0 \pm 1.3$ \\
\hline & & & & & 198 & 33.573 & 4.948 & 26.878 & $6.76 \pm 0.37$ & $1.85 \pm 0.10$ & $25.9 \pm 1.4$ \\
\hline & & & & & 297 & 33.658 & 3.589 & 27.022 & $5.43 \pm 0.36$ & $1.53 \pm 0.10$ & $21.3 \pm 1.4$ \\
\hline & & & & & 496 & 34.011 & 3.703 & 27.011 & $2.32 \pm 0.28$ & $0.96 \pm 0.08$ & $13.4 \pm 1.1$ \\
\hline & & & & & 692 & 34.247 & 3.506 & 27.030 & $2.61 \pm 0.25$ & $0.78 \pm 0.08$ & $10.9 \pm 1.1$ \\
\hline & $\mathrm{ES} 2$ & 2 Aug 2011 & $142^{\circ} 15^{\prime}$ & $37^{\circ} 04^{\prime}$ & 0 & & & & $11.02 \pm 0.48$ & $2.52 \pm 0.11$ & $35.2 \pm 1.5$ \\
\hline & & & & & 100 & 33.969 & 9.217 & 26.291 & $9.10 \pm 0.41$ & $2.44 \pm 0.11$ & $34.1 \pm 1.5$ \\
\hline & & & & & 199 & 34.095 & 7.752 & 26.515 & $4.39 \pm 0.24$ & $1.21 \pm 0.07$ & $16.9 \pm 0.9$ \\
\hline & & & & & 297 & 33.906 & 5.286 & 26.839 & $4.58 \pm 0.29$ & $1.28 \pm 0.08$ & $17.9 \pm 1.1$ \\
\hline & & & & & 496 & 34.148 & 4.571 & 26.920 & $2.86 \pm 0.19$ & $0.83 \pm 0.06$ & $11.6 \pm 0.8$ \\
\hline & & & & & 692 & 34.246 & 3.603 & 27.020 & $2.02 \pm 0.27$ & $0.59 \pm 0.08$ & $8.3 \pm 1.1$ \\
\hline & & & & & 989 & 34.400 & 2.864 & 27.089 & $1.94 \pm 0.26$ & $0.57 \pm 0.08$ & $8.0 \pm 1.1$ \\
\hline & & & & & 1978 & 34.591 & 1.862 & 27.171 & $0.48 \pm 0.15$ & $0.14 \pm 0.05$ & $2.0 \pm 0.6$ \\
\hline & FS2 & 2 Aug 2011 & $142^{\circ} 00^{\prime}$ & $37^{\circ} 00^{\prime}$ & 0 & & & & $11.02 \pm 0.48$ & $2.65 \pm 0.12$ & $37.0 \pm 1.6$ \\
\hline & & & & & 101 & 34.025 & 9.732 & 26.207 & $14.38 \pm 0.52$ & $2.91 \pm 0.10$ & $40.6 \pm 1.5$ \\
\hline & & & & & 197 & 33.770 & 6.585 & 26.677 & $7.62 \pm 0.45$ & $2.03 \pm 0.12$ & $28.4 \pm 1.7$ \\
\hline & & & & & 299 & 33.810 & 5.256 & 26.843 & $7.96 \pm 0.38$ & $2.18 \pm 0.10$ & $30.4 \pm 1.4$ \\
\hline & & & & & 497 & 33.985 & 3.523 & 27.028 & $4.77 \pm 0.22$ & $1.39 \pm 0.06$ & $19.4 \pm 0.9$ \\
\hline & & & & & 693 & 34.221 & 3.575 & 27.023 & $3.36 \pm 0.38$ & $0.98 \pm 0.11$ & $13.6 \pm 1.5$ \\
\hline & & & & & 990 & 34.372 & 3.017 & 27.075 & $1.38 \pm 0.18$ & $0.41 \pm 0.05$ & $5.7 \pm 0.7$ \\
\hline & & & & & 1307 & 34.480 & 2.420 & 27.127 & $1.09 \pm 0.19$ & $0.33 \pm 0.06$ & $4.6 \pm 0.8$ \\
\hline & FS5 & 3 Aug 2011 & $141^{\circ} 20^{\prime}$ & $36^{\circ} 00^{\prime}$ & 0 & & & & $99.62 \pm 2.12$ & $23.66 \pm 0.50$ & $330.9 \pm 7.0$ \\
\hline KT-11-27 & ST08 & 29 Oct 2011 & $142^{\circ} 00^{\prime}$ & $37^{\circ} 00^{\prime}$ & 0 & & & & $15.84 \pm 0.63$ & $4.33 \pm 0.17$ & $60.6 \pm 2.4$ \\
\hline & & & & & 49 & 33.881 & 17.928 & 24.515 & $11.62 \pm 0.42$ & $3.18 \pm 0.12$ & $44.5 \pm 1.6$ \\
\hline
\end{tabular}


Table 1. Continued.

\begin{tabular}{|c|c|c|c|c|c|c|c|c|c|c|c|}
\hline Cruise & Station & Date & E & $\mathrm{N}$ & $\begin{array}{c}\text { Depth } \\
\text { (m) }\end{array}$ & Salinity & $\begin{array}{c}\text { Potential } \\
\text { Temp. } \\
{ }^{\circ} \mathrm{C}\end{array}$ & Density & $\begin{array}{l}{ }^{129} \mathrm{I} /{ }^{127} \mathrm{I} \\
\times 10^{-11}\end{array}$ & $\times 10^{7}$ atoms $\mathrm{L}^{-1}$ & $\mathrm{nBqL}^{-1}$ \\
\hline & \multirow{13}{*}{$\mathrm{K} 8$} & \multirow{13}{*}{29 Oct 2011} & \multirow{13}{*}{$142^{\circ} 11^{\prime}$} & \multirow{13}{*}{$37^{\circ} 20^{\prime}$} & 100 & 34.135 & 11.199 & 25.951 & $10.83 \pm 0.50$ & $2.94 \pm 0.14$ & $41.2 \pm 1.9$ \\
\hline & & & & & 201 & 33.858 & 7.023 & 26.618 & $5.34 \pm 0.35$ & $1.50 \pm 0.10$ & $20.9 \pm 1.4$ \\
\hline & & & & & 300 & 33.773 & 4.542 & 26.923 & $4.09 \pm 0.27$ & $1.15 \pm 0.08$ & $16.1 \pm 1.1$ \\
\hline & & & & & 500 & 34.031 & 3.944 & 26.986 & $2.08 \pm 0.17$ & $0.60 \pm 0.05$ & $8.4 \pm 0.7$ \\
\hline & & & & & 1000 & 34.365 & 2.901 & 27.086 & $0.62 \pm 0.15$ & $0.19 \pm 0.05$ & $2.6 \pm 0.6$ \\
\hline & & & & & 0 & & & & $13.32 \pm 0.46$ & $3.61 \pm 0.12$ & $50.5 \pm 1.7$ \\
\hline & & & & & 49 & 34.194 & 17.051 & 24.726 & $9.38 \pm 0.53$ & $2.50 \pm 0.14$ & $35.0 \pm 2.0$ \\
\hline & & & & & 99 & 34.082 & 10.493 & 26.077 & $9.99 \pm 0.38$ & $2.75 \pm 0.11$ & $38.4 \pm 1.5$ \\
\hline & & & & & 201 & 33.706 & 5.621 & 26.799 & $4.41 \pm 0.24$ & $1.23 \pm 0.07$ & $17.3 \pm 0.9$ \\
\hline & & & & & 299 & 33.927 & 5.463 & 26.818 & $3.25 \pm 0.22$ & $0.92 \pm 0.06$ & $12.8 \pm 0.9$ \\
\hline & & & & & 500 & 34.093 & 4.278 & 26.952 & $1.71 \pm 0.15$ & $0.50 \pm 0.04$ & $7.0 \pm 0.6$ \\
\hline & & & & & & 34.231 & 3.469 & 27.033 & $0.92 \pm 0.13$ & $0.27 \pm 0.04$ & $3.8 \pm 0.6$ \\
\hline & & & & & 1020 & 34.394 & 2.719 & 27.101 & $0.22 \pm 0.15$ & $0.07 \pm 0.04$ & $0.9 \pm 0.6$ \\
\hline
\end{tabular}

at most stations after April 2011 except for two stations: station 8 during cruise KT-11-06 (28 April 2011) and station UW1 during cruise BO-11-05 (12 June 2011). Station $8\left(141^{\circ} 50^{\prime} \mathrm{E}, 36^{\circ} 31^{\prime} \mathrm{N}\right)$ is located near station UW1 $\left(141^{\circ} 53^{\prime} \mathrm{E}, 36^{\circ} 00^{\prime} \mathrm{N}\right)$. In the coastal regions, several water masses, such as the Kuroshio, meso-scale eddies associated with the Kuroshio, and fresh water from the land, coexist in a complex fashion. The satellite images (Ibaraki Prefectural Fisheries Experimental Station, 2012) of sea surface temperature indicate that the surface of station 8 at the time of observation was dominated by meso-scale eddies associated with the Kuroshio that were coming from low contamination regions (Buesseler et al., 2012). The lack of elevated ${ }^{129} \mathrm{I}$ concentrations at these two stations is considered to be caused by the complex seawater currents in the area near Fukushima.

The distribution patterns of surface ${ }^{131} \mathrm{I}$ and ${ }^{137} \mathrm{Cs}$ simulated numerically (Kawamura et al., 2011; Tsumune et al., 2012) showed that the accident-derived radionuclides supplied directly into the ocean were initially advected southward along the coast and then flowed eastward with the Kuroshio and its extension. The numerical simulation results from 25 March to 1 May 2011 also showed that the radionuclides were minimally advected northward near Fukushima (Kawamura et al., 2011). On the other hand, atmospheric dispersion simulations indicated that ${ }^{131} \mathrm{I}$ and ${ }^{137} \mathrm{Cs}$ were transported to the northeast and south of the 1FNPP and deposited on the surface of the western North Pacific (Kawamura et al., 2011; Terada et al., 2012). Thus, the observed accidentderived ${ }^{129} \mathrm{I}$ at northern stations can be inferred to be supplied to the ocean via atmospheric input. Another possibility mechanism to explain the high concentration at northern stations is the microbial volatilization at the southern stations. Released ${ }^{129}$ I to the ocean transported to southward with seawater current and then volatilized by microbial activity and then moved to northward by wind and then deposited.
Considering the ${ }^{129}$ I results, seawater current, and simulation results, we conclude that ${ }^{129} \mathrm{I}$ in the southern part from the 1FNPP was predominantly transported by seawater advection and that ${ }^{129} \mathrm{I}$ in the northern part of the 1FNPP was predominantly transported via the atmosphere including microbial volatilization.

\subsection{Depth profile of ${ }^{129} \mathrm{I}$}

The ${ }^{129}$ I concentrations in the seawater before and after the 1FNPP accident are plotted in Fig. 3 as a function of water depth. The all-depth profiles both before and after the 1FNPP accident show that the ${ }^{129}$ I concentration had its maximum in the surface-mixed layer and decreased with depth below the layer. Because there is no ${ }^{129} \mathrm{I}$ source in the Pacific Ocean, the differences in the ${ }^{129}$ I concentrations before and after the accident indicate the accident-derived ${ }^{129} \mathrm{I}$ in the water column. The ${ }^{129} \mathrm{I}$ concentrations after the accident at $1000 \mathrm{~m}$ depth were at levels similar to those before the accident. The results indicate that, as of October 2011, the accident-derived ${ }^{129}$ I spread mainly in the upper $100 \mathrm{~m}$ depth of this study area and that only a small amount was transported to deep layers. Inventories of ${ }^{129}$ I obtained in this study in the water column between the surface and $1000 \mathrm{~m}$ are summarized in Table 2 .

Integrated depth profiles show the inventory of ${ }^{129} \mathrm{I}$ including the background and the accident-derived ${ }^{129} \mathrm{I}$. The ocean inventories of ${ }^{129} \mathrm{I}$ depended on the distance from major reprocessing plants as well as water mass structures in the water column (Alfimov et al., 2004; Schink et al., 1995; Suzuki et al., 2010). Therefore, background inventories in the western margin of the North Pacific Ocean must be applied to evaluate the amount of ${ }^{129}$ I derived from the 1FNPP accident. Since accident-derived ${ }^{129} \mathrm{I}$ was not detected below $1000 \mathrm{~m}$ depth, the inventories for all profiles up to $1000 \mathrm{~m}$ are tabulated in Table 2 to evaluate the influence of the 1FNPP accident. Before the 1FNPP accident, the inventories in the upper $1000 \mathrm{~m}$ were in the $6.3-8.4 \times 10^{12}$ atoms $\mathrm{m}^{-2}$ range and averaged an inventory of $7.3 \times 10^{12}$ atoms $\mathrm{m}^{-2}$. 
Table 2. Inventory of ${ }^{129}$ I before and after the 1FNPP accident and its influence.

\begin{tabular}{|c|c|c|c|c|c|}
\hline Cruise & Station & $\begin{array}{l}\text { Sampling } \\
\text { date }\end{array}$ & $\begin{array}{c}\text { Inventory } \\
\left(\times 10^{12}{\left.\text { atoms } \mathrm{m}^{-2}\right)}^{-2}\right.\end{array}$ & $\begin{array}{c}\text { Increase after } \\
\text { the accident } \\
\left(\times 10^{12}{\left.\text { atoms } \mathrm{m}^{-2}\right)}^{-2}\right.\end{array}$ & Reference \\
\hline OS08 & KNOT & 10 May 2008 & 6.3 & & This study \\
\hline SY08 & off Kii & 5 Aug 2008 & 8.4 & & This study \\
\hline \multirow[t]{2}{*}{ SY09 } & Joban C & 18 Jul 2009 & 7.0 & & This study \\
\hline & Miyako & 20 Jul 2009 & 7.7 & & This study \\
\hline SY07 & Offshore of Kushiro & 1 Aug 2007 & 7.2 & & Suzuki et al. (2010) \\
\hline \multicolumn{2}{|c|}{ Average before the accident } & & 7.3 & & \\
\hline \multirow[t]{2}{*}{ KT-11-06 } & A & 29 Apr 2011 & 16.9 & 9.6 & This study \\
\hline & B & 30 Apr 2011 & 11.2 & 3.9 & This study \\
\hline \multirow[t]{3}{*}{ KH-11-07 } & FS1 & 2 Aug 2011 & 13.9 & 6.6 & This study \\
\hline & ES2 & 2 Aug 2011 & 10.8 & 3.5 & This study \\
\hline & FS2 & 2 Aug 2011 & 15.3 & 8.0 & This study \\
\hline \multirow[t]{2}{*}{ KT-11-27 } & ST08 & 29 Oct 2011 & 10.7 & 3.4 & This study \\
\hline & K8 & 29 Oct 2011 & 8.9 & 1.6 & This study \\
\hline
\end{tabular}

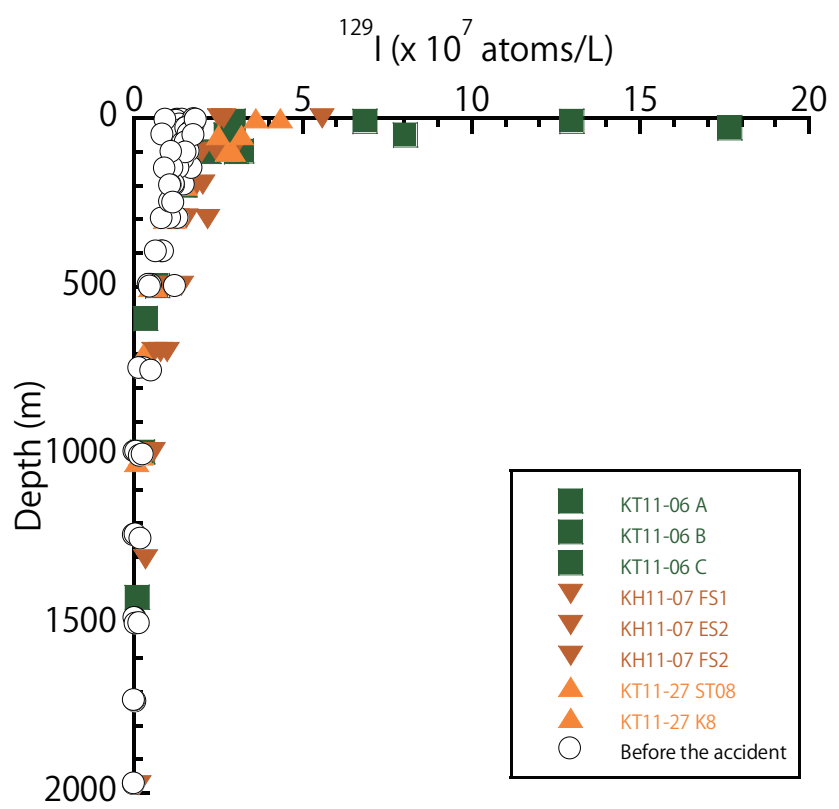

Fig. 3. Depth profiles of ${ }^{129} \mathrm{I}$ before and after the 1FNPP accident. The dark green, dark orange, and light orange symbols indicate cruises KT-11-06, KH11-07, and KT-11-27, respectively, after the 1FNPP accident. The white symbols indicate a cruise before the 1FNPP accident.

The inventories after the $1 \mathrm{FNPP}$ accident in the upper $1000 \mathrm{~m}$ were varied, $(8.9-16.9) \times 10^{12}$ atoms $\mathrm{m}^{-2}$. Inventories after the 1FNPP accident increased at all stations observed in this study. As a result of the accident, the increase in ${ }^{129} \mathrm{I}$ across

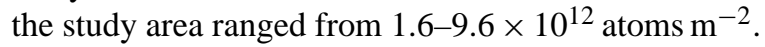

\subsection{Dose estimation from ${ }^{129} I$}

This study shows that the ${ }^{129}$ I concentration has increased near Fukushima on account of the 1FNPP accident. Because iodine is a biophilic element, ${ }^{129} \mathrm{I}$ enters the food chain and bio-accumulates in seafood. Because of its long half-life, an internal dose may be a serious concern for many generations. To evaluate the internal dose, an effective dose was roughly estimated based on ingestion of contaminated marine food. The effective dose can be obtained from the ${ }^{129} \mathrm{I}$ concentration in seawater $\left(\mathrm{Bq} \mathrm{L}^{-1}\right)$, the concentration factor for each marine food $\left(\mathrm{L} \mathrm{kg}^{-1}\right)$, the total amount of marine food consumed in a year, and the effective dose coefficient $\left({ }^{129} \mathrm{I}: 1.1 \times 10^{-7} \mathrm{~Sv} \mathrm{~Bq}^{-1}\right.$ for adults) (ICRP, 1995).

Radioiodine is accumulated in marine foods such as fish, crustaceans, mollusks, and macroalgae at concentration factors of $9,3,10$, and $10000 \mathrm{~L} \mathrm{~kg}^{-1}$, respectively (IAEA, 2004). The latest national survey revealed that the ordinary Japanese individual ingests $32.6 \mathrm{~g}$ fish, $4.4 \mathrm{~g}$ crustaceans, $2.9 \mathrm{~g}$ mollusks, and $11.0 \mathrm{~g}$ macroalgae in a day (MHLW, 2012). By assuming that the ${ }^{129}$ I concentrations in surface seawater after the 1FNPP accident continue to have the value of $1.08-89.8 \times 10^{7}$ atoms $\mathrm{L}^{-1}$ (corresponding to $15.2-$ $1255 \mathrm{nBq} \mathrm{L}^{-1}$ ) for a year, the effective dose was estimated to be $6.7-550 \times 10^{-11} \mathrm{~Sv} \mathrm{yr}^{-1}$. It was quite lower than the annual dose limit of $1.0 \times 10^{-3} \mathrm{~Sv} \mathrm{yr}^{-1}$. Since the ${ }^{129}$ I concentration in seawater near Fukushima is expected to decrease by dilution in the ocean, the actual effective dose would be much lower than the estimated one. Therefore, we concluded that the internal dose from the ingestion of seafood is negligibly small. 


\section{Conclusions}

This study focused on ${ }^{129} \mathrm{I}$, a long-lived radionuclide, derived from the 1FNPP accident. ${ }^{129}$ I concentrations in surface seawater before the 1FNPP accident, which can be used as the background level, showed a latitudinal gradient in horizontal distribution that could be expressed as a linear function of latitude between $32^{\circ} \mathrm{N}$ and $44^{\circ} \mathrm{N}$. In the western margin of the North Pacific, the highest and average ${ }^{129}$ I concentrations measured after the 1FNPP accident were 73 times and approximately 8 times, respectively, higher than those before the accident in this study area. The inventory of ${ }^{129} \mathrm{I}$ originating from the accident was $1.6-9.6 \times 10^{12}$ atoms $\mathrm{m}^{-2}$ in this study area.

Based on the conservative estimation from measurement data of ${ }^{129} \mathrm{I}$, the internal dose from the ingestion of seafood is negligibly small.

Because ${ }^{129} \mathrm{I}$ is one of the long-lived radionuclides, the data obtained in this study could be applied to the study of radionuclide migration from the 1FNPP accident. To investigate the impact of the 1FNPP accident more in detail, further investigation is required.

Acknowledgements. We deeply appreciate T. Gamo; M. Uematsu; H. Obata (Univ. of Tokyo); Y. Kato (Tokai Univ.); J. Zhang (Toyama Univ.); Y. W. Watanabe (Hokkaido Univ.); M. Minakawa (Fish. Res. Agcy., Japan); and the captains, crews, and researchers of the R/V Soyo Maru, T/S Oshoro Maru, R/V Tansei Maru, R/V Bosei Maru, and R/V Hakuho Maru for their assistance during sampling.

Edited by: H. Nies

\section{References}

Alfimov, V., Aldahan, A., Possnert, G., and Winsor, P.: Anthropogenic iodine-129 in seawater along a transect from the Norwegian coastal current to the North Pole, Mar. Pollut. Bull., 49, 1097-1104, 2004.

Baverstock, K., Egloff, B., Pinchera, A., Ruchera, C., Ruchti, C., and Williams, D.: Thyroid cancer after Chernobyl, Science, 359, 21-22, 1992.

Buesseler, K. O., Jayne, S. R., Fisher, N. S., Rypina, I. I., Baumann, H., Baumann, Z., Breier, C. F., Douglass, E. M., George, J., Macdonald, A. M., Miyamoto, H., Nishikawa, J., Pike, S. M., and Yoshida, S.: Fukushima-derived radionuclides in the ocean and biota off Japan, P. Natl. Acad. Sci. USA, 109, 5984-5988, 2012.

Campos, M. L. A. M.: New approach to evaluating dissolved iodine speciation in natural waters using cathodic stripping voltammetry and a storage study for preserving iodine species, Mar. Chem., 57, 107-117, 1997.

Honda, M. C., Aono, T., Aoyama, M., Hamajima, Y., Kawakami, H., Kitamura, M., Masumoto, Y., Miyazawa, Y., Takigawa, M., and Saino, T.: Dispersion of artifical cesium-134 and -137 in the western North Pacific one month after the Fukushima accident, Geochem. J., 44, e1-e9, 2012.
Hou, X. L., Dahlgaard, H., and Nielsen, S. P.: Chemical speciation analysis of ${ }^{129} \mathrm{I}$ in seawater and preliminary investigation to use it as a tracer for geochemical cycle study of stable iodine, Mar. Chem., 74, 145-155, 2001.

Hou, X. L., Povinec, P. P., Zhang, L., Shi, K., Biddulph, D., Chang, C. C. Fan, Y., Golser, R., Hou, Y., Ješkovský, M., Jull, A. J. T., Liu, Qi, Luo, M.,Steier, P., and Zhou, W.: Iodine-129 in seawater offshore Fukushima: Distribution, inorganic speciation, source, and budget, Environ. Sci. Technol., 47, 3091-3098, doi:10.1021/es304460k, 2013.

Ibaraki Prefectual Fisheries Experimental Station: http://www.pref. ibaraki.jp/bukyoku/nourin/suishi/gyomusen/noaa/old-noaa.htm, last access: 17 October 2012.

IAEA: Sediment distribution coefficients and concentration factors for biota in the marine environment, Vienna, 95, 2004.

ICRP: Age-dependent doses to the members of the public from intake of radionuclides - Part 5 compilation of ingestion and inhalation coefficients, ICRP Publication 72, 26, 1995.

Kawamura, H., Kobayashi, T., Furuno, A., In, T., Ishikawa, Y., Nakayama, T., Shima, S., and Awaji, T.: Preliminary numerical experiments on oceanic dispersion of ${ }^{131} \mathrm{I}$ and ${ }^{137} \mathrm{Cs}$ discharged into the ocean because of the Fukushima Daiichi Nuclear Power Plant disaster, J. Nucl. Sci. Technol., 48, 1349-1356, 2011.

Keogh, S. M., Aldahan, A., Possnert, G., Finegan, P., Vintro, L. L., and Mitchell, P. I.: Trends in the spatial and temporal distribution of ${ }^{129} \mathrm{I}$ and ${ }^{99} \mathrm{Tc}$ in coastal waters surrounding Ireland using Fucus vesiculosus as a bio-indicator, J. Environ. Radioact., 95, 23-38, 2007.

MHLW: The national health and nutrition survey in Japan, 2010, 195, 2012.

Michel, R., Daraoui, A., Gorny, M., Jakob, D., Sachse, R., Tosch, L., Nies, H., Goroncy, I., Herrmann, J., Synal, H. A., Stocker, M., and Alfimov, V.: Iodine-129 and iodine-127 in European seawaters and in precipitation from Northern Germany, Sci. Total Environ., 419, 151-169, 2012.

Miyake, Y., Matsuzaki, H., Fujiwara, T., Saito, T., Yamagata, T., Honda, M., and Muramatsu, Y.: Isotopic ratio of radioactive iodine $\left({ }^{129} \mathrm{I} /{ }^{131} \mathrm{I}\right)$ released from Fukushima Daiichi NPP accident, Geochem. J., 46, 327-333, 2012.

Momoshima, N., Sugihara, S., Ichikawa, R., and Yokoyama, H.: Atmospheric radionuclides transported to Fukuoka, Japan remote from the Fukushima Dai-ichi nuclear power complex following the nuclear accident, J. Environ. Radioact., 111, 28-32, 2012.

Moran, J. E., Oktay, S., Santschi, P. H., and Schink, D. R.: Atomospheric dispersal of ${ }^{129}$ iodine from nuclear fuel reprocessing facilities, Environ. Sci. Technol., 33, 2536-2542, 1999.

Muramatsu, Y., Yoshida, S., Fehn, U., Amachi, S., and Ohmomo, Y.: Studies with natural and anthropogenic iodine isotopes: iodine distribution and cycling in the global environment, J. Environ. Radioact., 74, 221-232, 2004.

Povinec, P. P., Lee, S. H., Kwong, L. L. W., Oregioni, B., Jull, A. J. T., Kieser, W. E., Morgenstern, U., and Top, Z.: Tritium, radiocarbon, ${ }^{90} \mathrm{Sr}$ and ${ }^{129} \mathrm{I}$ in the Pacific and Indian Oceans, Nucl. Instrum. Meth. B, 268, 1214-1218, 2010.

Raisbeck, G. M. and Yiou, F.: ${ }^{129}$ I in the oceans: origins and applications, Sci. Total Environ., 237/238, 31-41, 1999.

Schink, D. R., Santschi, P. H., Corapcioglu, O., Sharma, P., and Fehn, U.: ${ }^{129}$ I in Gulf of Mexico waters, Earth. Planet. Sci. Lett., 135, 131-138, 1995. 
Snyder, G., Aldahan, A., and Possnert, G.: Global distribution and long-term fate of anthropogenic ${ }^{129} \mathrm{I}$ in marine and surface water reservoirs, Geochem. Geophy. Geosy., 11, Q04010, doi:10.1029/2009GC002910, 2010.

Suzuki, T., Kitamura, T., Kabuto, S., Togawa, O., and Amano, H.: High sensitivity measurement of iodine-129/iodine-127 ratio by accelerator mass spectrometry, J. Nucl. Sci. Technol., 43, 14311435, 2006.

Suzuki, T., Kabuto, S., Amano, H., and Togawa, O.: Measurement of iodine-129 in seawater samples collected from the Japan Sea area using accelerator mass spectrometry: Contribution of nuclear fuel reprocessing plants, Quat. Geochronol., 3, 268-275, 2008.

Suzuki, T., Minakawa, M., Amano, H., and Togawa, O.: The vertical profiles of iodine-129 in the Pacific Ocean and the Japan Sea before the routine operation of new nuclear fuel reprocessing plant, Nucl. Instrum. Meth. B, 268, 1229-1231, 2010.
Terada, H., Katata, G., Chino, M., and Nagai, H.: Atmospheric discharge and dispersion of radionuclides during the Fukushima Dai-ichi Nuclear Power Plant accident. Part II: verification of the source term and analysis of regional-scale atmospheric dispersion, J. Environ. Radioact., 112, 141-154, 2012.

Toyama, C., Muramatsu, Y., Uchida, Y., Igarashi, Y., Aoyama, M., and Matsuzaki, H.: Variations of ${ }^{129} \mathrm{I}$ in the atmospheric fallout of Tokyo, Japan: 1963-2003, J. Environ. Radioact., 113, 116122, 2012.

Tsumune, D., Tsubono, T., Aoyama, M., and Hirose, K.: Distribution of oceanic ${ }^{137} \mathrm{Cs}$ from the Fukushima Dai-ichi Nuclear Power Plant simulated numerically by a regional ocean model, J. Environ. Radioact., 111, 100-108, 2012. 